\title{
Antemortem diagnosis of a ventricular septal defect in a California sea lion Zalophus californianus
}

\author{
Sophie E. Dennison ${ }^{1,2, *}$, William Van Bonn ${ }^{2}$, Marjorie Boor ${ }^{2,3}$, James Adams ${ }^{3}$, \\ Nicola Pussini ${ }^{2}$, Terry Spraker ${ }^{4}$, Frances M. D. Gulland ${ }^{2}$ \\ ${ }^{1}$ Consultant Veterinary Radiologist, Marine Mammal Radiology, San Francisco, California 94107, USA \\ ${ }^{2}$ The Marine Mammal Center, 2000 Bunker Road, Sausalito, California 94965, USA \\ ${ }^{3}$ Cardiovascular Associates of Marin and San Francisco, Larkspur, California 94939, USA \\ ${ }^{4}$ Colorado State University Diagnostic Laboratory, College of Veterinary Medicine and Biomedical Sciences, \\ Colorado State University, Fort Collins, Colorado 80526, USA
}

\begin{abstract}
A yearling California sea lion Zalophus californianus stranded in poor body condition, and on physical examination a heart murmur was audible bilaterally. The sea lion was diagnosed with a left-to-right shunting membranous ventricular septal defect (VSD) using B-mode, color-flow Doppler and continuous-wave Doppler echocardiography. A left-to-right intracardiac shunting lesion was confirmed during cardiac angiographic computed tomography. The VSD defect was verified during the necropsy examination. On histologic examination concurrent mild multifocal myocarditis with focal mild ventricular free-wall myocardial necrosis were identified. A specific cause for the myocarditis and myocardial necrosis was not found, and association with the VSD and resultant myocardial dysfunction was presumed. This is the first report of the antemortem diagnosis of a VSD in a marine mammal and the first report of a VSD in a California sea lion.
\end{abstract}

KEY WORDS: Echocardiography $\cdot$ Congenital defect $\cdot$ Cardiac disease $\cdot$ Heart disease $\cdot$ Ultrasound

\section{INTRODUCTION}

A 31 kg, 117 cm standard length, male yearling California sea lion Zalophus californianus stranded and was presented to The Marine Mammal Center (TMMC, Sausalito, California) for health assessment and appropriate veterinary care. At presentation, the animal was moderately underweight and lethargic; on auscultation, a grade V/VI systolic heart murmur was detected, the point of maximal intensity being equal on both sides of the thorax at the heart base. The animal's mucous membranes were pale pink with normal capillary refill time $(<2 \mathrm{~s})$. Abdominal palpation and the results of the hematology and serum chemistry profile were unremarkable.

\section{EXAMINATION, RESULTS AND DISCUSSION}

The animal was manually restrained, and anesthesia was induced by utilizing a mask with isoflurane in $100 \%$ oxygen. Ventilation was spontaneous throughout the imaging period. Following endotracheal intubation, echocardiography was performed utilizing B-mode, color-flow Doppler and continuous-wave Doppler (7 $\mathrm{MHz}$ broadband phased-array transducer, Acuson X300, Siemens Healthcare). The echocardiogram demonstrated a defect high in the ventricular septum (Fig. 1) immediately ventral to the tricuspid and aortic valves within the membranous interventricular septum and consistent with a ventricular septal defect (VSD). The defect measured $\sim 1 \mathrm{~cm}$. The shunt was determined 


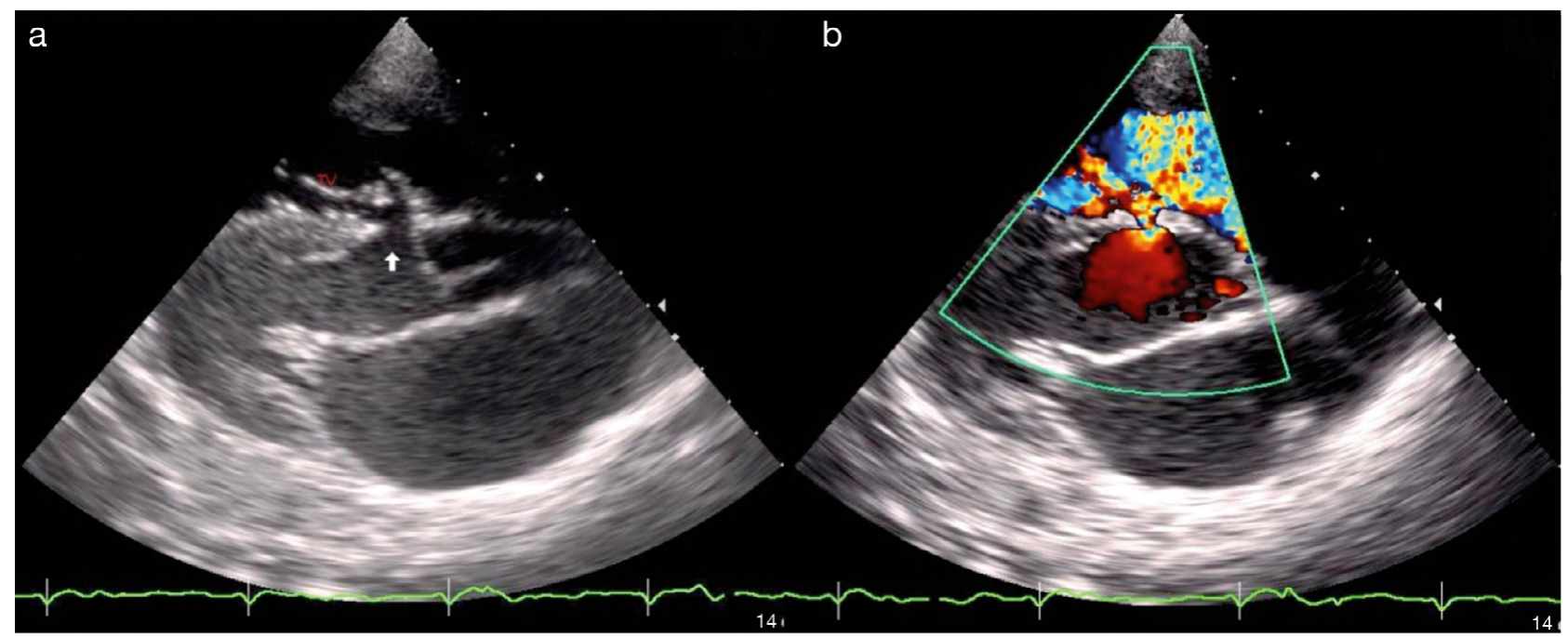

Fig. 1. Zalophus californianus. (a) B-mode ultrasound image taken from a right parasternal approach. White arrow points to the defect in the interventricular septum. The right ventricle is above the defect and the left ventricle below. The right side of the heart is moderately dilated due to volume overload. (b) Color-flow Doppler of the ventricular septal defect (VSD). Red flow from the left ventricle through the defect is towards the transducer and this, in combination with turbulence (multiple colors) in the right ventricle, indicates a left-to-right shunt

to be left-to-right using color-flow Doppler (Fig. 1) and continuous-wave Doppler. Both ventricles were subjectively dilated. Other cardiac structures were considered normal. Fractional shortening was calculated as $14.5 \%$ and ejection fraction as $31 \%$. Heart rate was considered normal at 90 beats per minute (bpm). Published normal cardiac values for fractional shortening and ejection fraction for this species have not been published to the authors' knowledge.

On observation of those echocardiogram findings, we obtained right lateral and ventro-dorsal radiographs of the thorax. Radiographs showed an enlarged cardiac silhouette with a calculated vertebral heart score (VHS) from the lateral radiographic view of 10.5 compared to the previously reported mean VHS of 8.65 (Dennison et al. 2009), and increased width on the ventro-dorsal view with the cardiac silhouette occupying $78 \%$ compared to the previous mean normal reported as $66 \%$ (Dennison et al. 2009). The pulmonary arteries and veins were symmetrical and enlarged compared to radiographs of other animals without evidence of cardiovascular disease, consistent with pulmonary over-circulation. A mixed bronchointerstitial to alveolar pattern was identified in all lung fields and was more apparent ventrally. These radiographic findings were most consistent with a left-toright shunting VSD and concurrent pneumonia and/or early cardiogenic pulmonary edema. The ventral distribution and lack of pulmonary venous distension was more consistent with pneumonia (aspiration, verminous or bacterial).
Recovery from anesthesia was uneventful. Therapy for verminous pneumonia was started; it consisted of penicillin G procaine/penicillin G benzathine $1 \mathrm{ml}$ per $10 \mathrm{~kg}$ intramuscular (i.m.) every other day (150000 units of both penicillin forms in suspension; Pen BP-48, IVX Animal Health), carprofen $4.4 \mathrm{mg} \mathrm{kg}^{-1}$ once daily orally (100 mg chewable tablets; Rimadyl, Pfizer Animal Health) and 1 dose of ivermectin $200 \mu \mathrm{kg}^{-1}$ i.m. (1\% injectable formula; Ivomec, Merial) on Day 3 of the carprofen therapy. No specific cardiac medications were initiated.

Three weeks later the sea lion was clinically similar and had progressively lost weight. A non-selective cardiac angiographic computed tomography (CT) study was performed to gather more clinical information and to determine the feasibility of this study in this species. Following induction of anesthesia and endotracheal intubation, a catheter was placed in the right subclavian vein under ultrasound guidance. The tip of the catheter was at the level of the right atrium. The sea lion was positioned in a slightly obliqued dorsally recumbent position to permit access to the subclavian catheter without repositioning between scans. The scan was acquired using a 64-slice multi-row detector CT scanner (Lightspeed VCT, GE Healthcare). Survey series of the thorax were obtained using soft-tissue and bone (medium- and high-frequency) reconstruction algorithms. The non-selective cardiac angiographic CT was acquired as a cine loop with a slice thickness of $5 \mathrm{~mm}$, equivalent single slice scanner pitch of 1.5 and a technique of 120 kilovolt peak (KVp) and $100 \mathrm{~mA}$. The 
scan was initiated simultaneously with the administration of a bolus of $60 \mathrm{ml}$ of $300 \mathrm{mgI} \mathrm{ml}^{-1}$ non-ionic iodinated contrast medium (iohexol, Omnipaque, GE Healthcare) via the subclavian venous catheter, and continued until images of both the dextro- and levophases of the passage of the contrast medium through the heart had been acquired. A soft-tissue reconstruction algorithm was applied to the angiographic data. The non-selective cardiac angiographic CT (Fig. 2) confirmed left-to-right shunting by the absence of premature opacification of the left ventricle during the dextrophase followed by persistent rightsided opacification. Aorta opacification occurred simultaneously with the left ventricle. The persistent opacification of the right side of the heart had attenuation values identical to the left side of the heart, and the attenuation on both sides diminished simultaneously and equally through sequential cardiac cycles. These findings indicated recirculation (shunting) from the left, but the exact location of the shunt was not observed. The pulmonary lobar arteries and veins could be only partially evaluated during the angiographic study in the region scanned and were symmetric in diameter. An alveolar pattern was identified bilaterally within the dorsal lung lobes and was worse on the right side. There was an associated rightward mediastinal shift. An interstitial to patchy alveolar pattern was identified in the inflated (mid and ventral) lung lobes. The dorsal alveolar pattern with a mediastinal shift was most consistent with positional atelectasis. The changes in the inflated lung suggested that pulmonary pathology was present, differential diagnoses for which included pneumonia or pulmonary edema, or both. Neoplasia was not considered likely given the age of the patient and the low frequency with which pulmonary neoplasia is identified in this species.

Due to poor prognosis, the sea lion was humanely euthanized following the procedure. A necropsy examination was performed with representative samples of each tissue and the entire heart was fixed in $5 \%$ formalin for histology. At necropsy, the presence of a membranous VSD was verified, and right and left ventricular dilatations were observed. The ventricular wall measurements were within normal limits. No other congenital cardiac or non-cardiac malformations were identified. The lungs were firm, heavy, wet and congested throughout all lobes. Fluid oozed from the surface of cuts made into the lung lobes. The liver had a mild reticulated pattern and was friable. The causes of death recorded following the necropsy examination were euthanasia due to VSD, verminous pneumonia and possible congestive heart failure. Histology demonstrated multifocal regions of lymphocyte infiltration with scant plasma cells within the myocardium of the left and right ventricular free walls. Similar mild changes were identified in the interventricular septum and walls of both atria. In one focal area of left ventricular free wall examined there was also evidence of mild myocardial necrosis. The myocarditis was considered multifocal, mild and subacute. A solitary cyst of
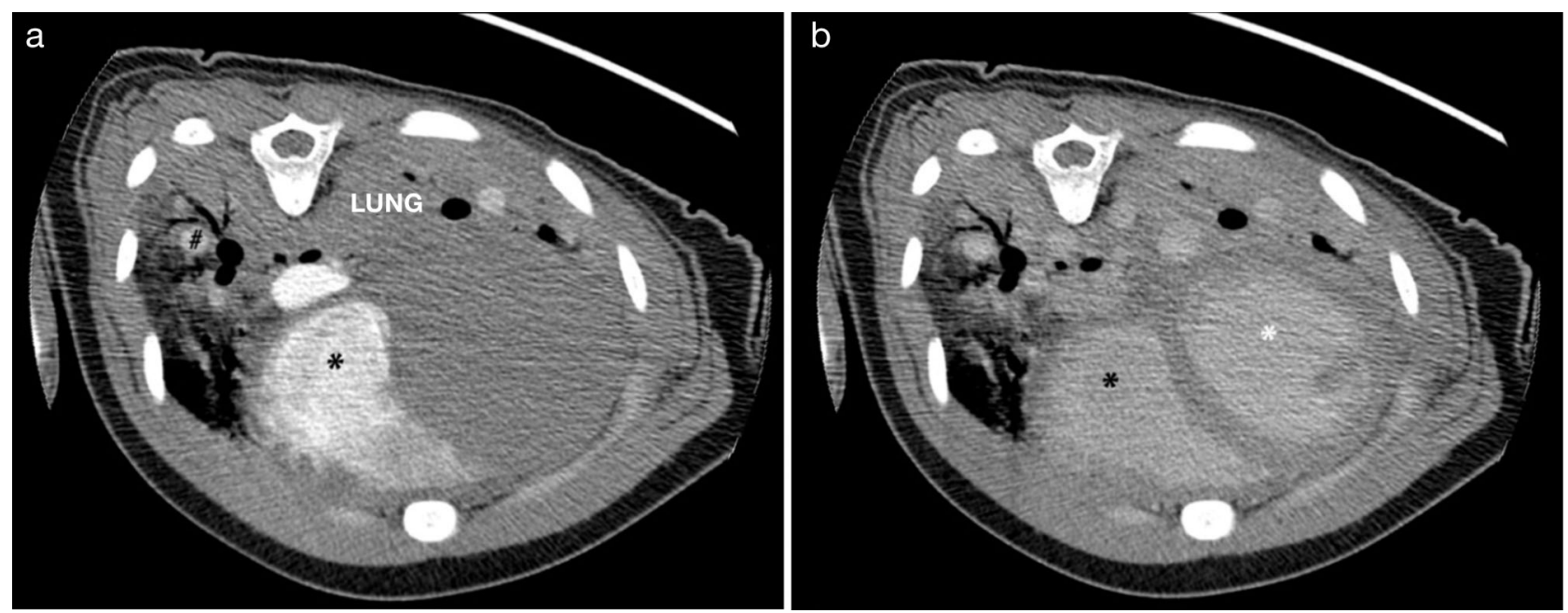

Fig. 2. Zalophus californianus. Transverse computed tomography (CT) image through the heart during non-selective angiography acquired using a medium-frequency algorithm and displayed at WW414, WL143. The patient's right side is to the left of the image. Curved line in the top right corner of the images is the cradle in which the sea lion was positioned for the scan. (a) During the dextrophase there is opacification of the right ventricle (black *) and left and right (\#) pulmonary arterial branches. There is no evidence of abnormal opacification of the left ventricle, ruling out any evidence of right-to-left shunting. The left dorsal lung (LUNG) increased in attenuation, which may be due to disease or atelectasis. The actual vetricular septal defect cannot be seen in this image. (b) During the levophase there is now opacification of the left ventricle (white *) and persistent, similarly attenuating, opacification of the right ventricle (black *) and pulmonary vasculature, indicating that a left-to-right shunt is occurring. During the levophase more vascular structures are seen as both the pulmonary veins and the pulmonary arteries are opacified 
Sarcocystis sp., without associated inflammation, was identified among the myocardial tissues examined. Myocardial fibrosis and mineralization were not identified. Special stains, including Gomori methanaminesilver, Kinyoun acid-fast stain and Brown \& Brenn Gram stain, were used to attempt to determine the etiology of the myocarditis, but results were negative. Culture of fresh tissue was not performed as the entire heart was formalin-fixed for histology. The sections of lung examined showed alveolar lobular edema and capillary congestion consistent with subacute pulmonary edema and also regions with fibrin, numerous macrophages, neutrophils, plasma cells and lymphocytes with exudate extending into some terminal bronchioles consistent with chronic pneumonia. The distribution and degree of pulmonary edema observed in association with the verminous pneumonia was no different to that seen in other sea lions without evidence of cardiac disease. Larval Parafilaroides decorus were identified in association with the regions of suppurative inflammation, but adult worms were not found. The hepatic lesions were characterized by a few multifocal accumulations of macrophages, lymphocytes and plasma cells with mild Kupffer cell hyperplasia consistent with parasitic migration and were considered incidental. Mild centrilobular fatty degeneration associated with malnutrition was also found, but hepatic congestion was not identified. The cause of death was recorded as euthanasia due to failure to thrive, and this was attributed to the persistent pulmonary pathology and presence of the VSD. The myocarditis was mild and considered a consequence of the VSD pathology after ruling out other etiologies.

Following the reported histology findings, paired banked sera were submitted to the University of California, Davis, Department of Pathology, Microbiology and Immunology for serology for Toxoplasma sp. and Sarcocystis neurona using an immunofluorescent antibody technique (IFAT). The serology (IFAT) results showed an antibody titer of 1:160 against Toxoplasma sp. and $<1: 40$ against $S$. neurona. Both results were interpreted as too low to indicate active infection.

Retrospectively, the echocardiogram findings and calculated values were compared to data that had recently been collected from 5 clinically healthy, captive-maintained, adult California sea lions. Fractional shortening for this VSD case was lower than the mean for the clinically healthy animals (14.5 vs. $37.6 \%$, range 36 to $40 \%$ ), as was ejection fraction (31 vs. $67.2 \%$, range 62 to $70 \%$ ). Heart rate was similar (90 vs. $88.2 \mathrm{bpm}$, range 80 to $95 \mathrm{bpm}$ ).

VSDs have sporadically been identified at necropsy in stranded northern elephant seals (Trupkiewicz et al. 1997, Colegrove et al. 2005) and in a bottlenose dolphin (Powell et al. 2009), and the clinical significance in those cases was unclear. Four cases of VSD in northern elephant seal have been reported, and at least one other congenital abnormality was identified in every case, including hydrocephalus, patent ductus arteriosus (PDA) (Colegrove et al. 2005), partial aortic coarctation and pulmonary arterial aplasia with right ventricular hypoplasia (Trupkiewicz et al. 1997). Other published cardiovascular congenital anomalies in marine mammal species describe lesions that have been identified during necropsy examination, and these include a persistent right aortic arch (PRAA) in a northern elephant seal (MacLean et al. 2008), an atrial septal defect (ASD) in a common dolphin Delphinus delphis (Jepson et al. 2005) and multiple abnormalities, including VSD, ASD, mitral valve and pulmonary artery hypoplasia with associated secondary changes, in a bottlenose dolphin Tursiops truncatus (Powell et al. 2009). No publications could be found that describe the antemortem diagnosis of cardiovascular congenital defects in any marine mammal.

VSDs are categorized as membranous or muscular depending on the location within the interventricular septum. The etiology of VSD is poorly understood but the defect occurs during embryologic development when there is failure in fusion of the right and left bulbar ridges, the endocardial cushion and the muscular interventricular septum (Kienle \& Kittleson 1998). Such membranous defects, although infrequently observed in small animal medicine, are diagnosed more commonly than muscular VSDs (Kittleson 1998a). Moderate to large defects typically result in significant leftto-right shunting during systole, with the majority of the shunting blood effectively bypassing the right side of the heart and directly entering the pulmonary circulation before returning to the left side of the heart. This results in pulmonary overcirculation and left-sided cardiac volume overload. Right-sided changes most frequently occur when chronic pulmonary overcirculation results in pulmonary hypertension. While the right side of the heart in this case was subjectively enlarged, we found no evidence to support pulmonary hypertension, at least while at sea level and in a non-diving state. It is possible that shunt reversal or other hemodynamic consequence occurred during dives to forage, limiting food consumption and resulting in the underweight presentation of the patient. However, many animals of this age and size in a similar state of malnourishment (low body weight compared to body length) present every year to TMMC without evidence of cardiac disease (Greig 2005).

The echocardiogram produced from this sea lion permitted detection of the VSD and confirmation that shunting was left-to-right using color-flow and continuous-wave Doppler. Echocardiography is a noninvasive and relatively inexpensive method of real- 
time cardiac imaging. Within the field of marine mammal medicine its use has been described in captive Tursiops truncatus (Sklansky et al. 2006), but descriptions in other marine mammal species are lacking.

In small animal medicine, radiographs are reliably used to determine whether or not pulmonary edema is present. A finding of interstitial to alveolar pattern in dogs, or bronchial to alveolar pattern in cats, with concurrent pulmonary venous congestion and cardiomegaly are strongly suggestive of left-sided congestive heart failure. Although the etiology remains uncertain, pulmonary edema was present in this sea lion at the time of death. Congestive heart failure was not diagnosed from radiographs or CT due to interpretation of the symmetrically enlarged pulmonary arteries and veins being pulmonary overcirculation without pulmonary venous congestion. Tachycardia, which is common in terrestrial mammals in left-sided congestive heart failure, was never observed in this sea lion. The absence of pulmonary venous congestion and tachycardia may be a species-specific variation that one should be aware of when managing sea lion cardiac disease. However, evidence of erythrophagocytosis that would have further indicated congestive heart failure on histology was not observed, and the fibrin and degree and distribution of the edema was not considered any different to histologic review of other sea lions treated for chronic verminous pneumonia without cardiac disease. It is quite feasible that acute cardiogenic edema may have been masked by verminous pneumonia-associated edema. Far more work is needed to characterize the imaging and clinical findings associated with congestive heart failure antemortem to determine features that permit differentiation from pneumonia in this species.

The concurrent mild myocarditis was an unexpected histologic finding. It was identified multifocally in the left and right ventricular free walls, interventricular septum and atrial walls but was unrelated to the conducting system. Myocarditis in southern sea otters has been associated with domoic acid toxicosis and Sarcocystis neurona infection (Kreuder et al. 2005). In the pygmy and dwarf sperm whales, right-sided congestive heart failure with pale right ventricles with extensive myocellular degeneration, atrophy and fibrosis were identified histologically. In those cases an etiology for the myocarditis was not identified (Bossart et al. 2007). California sea lions suffering from the acute and long-term effects of domoic acid toxicosis have also shown histologic lesions that were characterized by mixed-age acute to chronic, mild to severe foci of myocardial degeneration and necrosis with a specific distribution along the conducting system (Zabka et al. 2009). Domoic acid toxicosis was not considered a likely etiology in this case due to distribution and lack of associated brain lesions. Toxoplasma sp. and Sarcocystis neurona titers were not interpreted as significant and there was no inflammation associated with the solitary cyst identified during histology. Special stains were negative and failed to provide additional information. Concurrent bacterial pneumonia in human cardiac patients has been an accepted etiology for myocarditis since the 1940s (Saphir \& Amromin 1948), but in small animal medicine, hematogenous spread of septic (usually bacterial, rarely fungal) emboli from pneumonia usually results in endocarditis with vegetative lesions adhered to the mitral and aortic valves (Kittleson 1998b). Multifocal necrotizing myopathy and cardiomyopathy has been described as a cause of death in pups of the northern fur seal Callorhinus ursinus (Spraker \& Lander 2010) and affects the left ventricular, and rarely, the right ventricular free walls. The etiology is unknown at this time. Fungal myocarditis has been infrequently described in small animal medicine (Kittleson 1998b). Suppurative inflammation and infectious agents would have been expected if this were a feasible etiology but were not identified during histology of the myocardium or lungs in this case. Thus, the most likely cause for the inflammation and necrosis in this case is hypothesized as myocardial damage due to volume overload from the VSD.

In conclusion we describe the first antemortem diagnosis of a left-to-right shunting VSD in a California sea lion using echocardiography. Cardiac angiographic CT also demonstrated the presence of a shunt but the exact location was not identified. CT permits simultaneous evaluation of the lungs, but in this case significant additional information was not obtained due to the presence of verminous pneumonia. Evaluation for cardiac congenital defects should be performed at the earliest opportunity when cardiac murmurs or cardiomegaly are identified in young animals undergoing rehabilitation.

\section{LITERATURE CITED}

Bossart GD, Hensley G, Goldstein JD, Kroell K, Manire CA, Defran RH, Reif JS (2007) Cardiomyopathy and myocardial degeneration in stranded pygmy (Kogia breviceps) and dwarf (Kogia sima) sperm whales. Aquat Mamm 33: 214-222

Colegrove KM, Greig DJ, Gulland FMD (2005) Causes of live strandings of northern elephant seals (Mirounga angustirostris) and Pacific harbor seals (Phoca vitulina) along the central California coast, 1992-2001. Aquat Mamm 31: $1-10$

> Dennison SE, Forrest L, Gulland FMD (2009) Normal thoracic radiographic anatomy of immature California sea lions (Zalophus californianus) and immature northern elephant seals (Mirounga angustirostris). Aquat Mamm 35:36-42

> Greig DJ, Gulland FMD, Kreuder C (2005) A decade of live California sea lion (Zalophus californianus) strandings 
along the central California coast: causes and trends, 1991-2000. Aquat Mamm 31:11-22

Jepson PD, Deaville R, Patterson IAP, Pocknell AM and others (2005) Acute and chronic gas bubble lesions in cetaceans stranded in the UK. Vet Pathol 42:291-305

Kienle RD, Kittleson MD (1998) Embryology and anatomy. In: Kittleson MD, Kienle RD (eds) Small animal cardiology, Chap 1. Mosby, Maryland Heights, MO, p 1-10

Kittleson MD (1998a) Septal defects. In: Kittleson M D, Kienle RD (eds) Small animal cardiology, Chap 13. Mosby, Maryland Heights, MO, p 231-239

Kittleson MD (1998b) Infective endocarditis. Kittleson MD, Kienle RD (eds) Small animal cardiology, Chap 24. Mosby, Mryland Heights, MO, p 402-412

Kreuder C, Miller MA, Lowenstine LJ, Conrad PA, Carpenter TE, Jessup DA, Mazet JAK (2005). Evaluation of cardiac lesions and risk factors associated with myocarditis and dilated cardiomyopathy in southern sea otters (Enhydra lutris nereis). Am J Vet Res 66:289-299

MacLean RA, Imai D, Dold C, Haulena M, Gulland FMD (2008) Persistent right aortic arch and cribriform plate aplasia in a northern elephant seal (Mirounga angusti-

Editorial responsibility: John Austin, Oldendorf/Luhe, Germany rostris). J Wildl Dis 44:499-504

> Powell JWB, Archibald RT, Cross CA, Rotstein DS, Soop VM (2009) Multiple congenital cardiac abnormalities in an Atlantic bottlenose dolphin (Tursiops truncatus). J Wildl Dis 45:839-842

Saphir O, Amromin GD (1948) Myocarditis in instances of pneumonia. Ann Intern Med 28:963-970

Sklansky M, Levine G, West N, Renner M, Rimmerman C, Stone R (2006) Echocardiographic evaluation of the bottlenose dolphin (Tursiops truncatus). J Zoo Wildl Med 37:454-463

Spraker TR, Lander ME (2010) Causes of mortality in northern fur seals (Callorhinus ursinus), St. Paul Island, Pribilof Islands, Alaska, 1986-2006. J Wildl Dis 46:450-473

Trupkiewicz JG, Gulland FM, Lowenstine LJ (1997) Congenital defects in northern elephant seals stranded along the central California coast. J Wildl Dis 33:220-225

Zabka TS, Goldstein T, Cross C, Mueller RW, KreuderJohnson C, Gill S, Gulland FMD (2009) Characterization of a degenerative cardiomyopathy associated with domoic acid toxicity in California sea lions (Zalophus californianus). Vet Pathol 46:105-119

Submitted: June 30, 2010; Accepted: November 29, 2010

Proofs received from author(s): February 28, 2011 